\title{
A comparison of ear-canal-reflectance measurement methods in an ear simulator
}

\author{
Nørgaard, Kren Rahbek; Charaziak, Karolina K. ; Shera, Christopher A.
}

Published in:

Journal of the Acoustical Society of America

Link to article, DOI:

$10.1121 / 1.5123379$

Publication date:

2019

Document Version

Publisher's PDF, also known as Version of record

Link back to DTU Orbit

Citation (APA):

Nørgaard, K. R., Charaziak, K. K., \& Shera, C. A. (2019). A comparison of ear-canal-reflectance measurement methods in an ear simulator. Journal of the Acoustical Society of America, 146(2), 1350-1361.

https://doi.org/10.1121/1.5123379

\section{General rights}

Copyright and moral rights for the publications made accessible in the public portal are retained by the authors and/or other copyright owners and it is a condition of accessing publications that users recognise and abide by the legal requirements associated with these rights.

- Users may download and print one copy of any publication from the public portal for the purpose of private study or research.

- You may not further distribute the material or use it for any profit-making activity or commercial gain

- You may freely distribute the URL identifying the publication in the public portal

If you believe that this document breaches copyright please contact us providing details, and we will remove access to the work immediately and investigate your claim. 


\title{
A comparison of ear-canal-reflectance measurement methods in an ear simulator
}

\author{
Kren Rahbek Nørgaard ${ }^{\text {a) }}$ \\ Acoustic Technology, Department of Electrical Engineering, Technical University of Denmark, Ørsteds Plads, \\ Building 352, Kongens Lyngby, DK-2800, Denmark

\begin{abstract}
Karolina K. Charaziak and Christopher A. Shera ${ }^{\text {b) }}$
Caruso Department of Otolaryngology, University of Southern California, 1640 Marengo Street, Los Angeles, California 90033, USA
\end{abstract}

(Received 31 May 2019; revised 2 August 2019; accepted 3 August 2019; published online 23 August 2019)

Ear-canal reflectance has been researched extensively for diagnosing conductive hearing disorders and compensating for the ear-canal acoustics in non-invasive measurements of the auditory system. Little emphasis, however, has been placed on assessing measurement accuracy and variability. In this paper, a number of ear-canal-reflectance measurement methods reported in the literature are utilized and compared. Measurement variation seems to arise chiefly from three factors: the residual ear-canal length, the ear-probe insertion angle, and the measurement frequency bandwidth. Calculation of the ear-canal reflectance from the measured ear-canal impedance requires estimating the ear-canal characteristic impedance in situ. The variability in ear-canal estimated characteristic impedance and reflectance due to these principal factors is assessed in an idealized controlled setup using a uniform occluded-ear simulator. In addition, the influence of this measurement variability on reflectance-based methods for calibrating stimulus levels is evaluated and, by operating the condenser microphone of the occluded-ear simulator as an electro-static speaker, the variability in estimating the emitted pressure from the ear is determined. The various measurement methods differ widely in their robustness to variations in the three principal factors influencing the accuracy and variability of ear-canal reflectance. (C) 2019 Acoustical Society of America.

https://doi.org/10.1121/1.5123379

[BLM]

Pages: 1350-1361

\section{INTRODUCTION}

The measurement of ear-canal reflectance is useful for a number of hearing-diagnostic applications. Evidence suggests that the ear-canal reflectance can be used directly as a tool to diagnose and distinguish different conductive hearing disorders (Ellison et al., 2012; Feeney and Keefe, 2001; Keefe et al., 2000; Keefe et al., 2012; Merchant et al., 2014; Piskorski et al., 1999) because it represents the delayed reflection from the tympanic membrane in a uniform ear canal. For behavioral auditory thresholds and in measurements of evoked responses in the ear canal, it is useful for calibrating stimulus levels (Lewis et al., 2009; McCreery et al., 2009; Scheperle et al., 2011; Scheperle et al., 2008; Souza et al., 2014; Withnell et al., 2009). Finally, for acoustic responses elicited in the inner ear and measured in the ear canal, e.g., otoacoustic emissions (OAE), it can be used for estimating the sound pressure emitted from the tympanic membrane into an anechoic ear canal (Charaziak and Shera, 2017).

The ear-canal impedance is usually measured using an insert ear probe following a preliminary calibration procedure to obtain its acoustic Thévenin-equivalent source

\footnotetext{
a) Also at: Interacoustics A/S, Audiometer Allé 1, Middelfart, DK-5500, Denmark. Electronic mail: krng@interacoustics.com

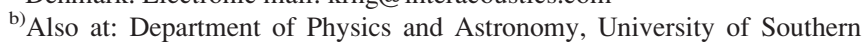
California, Los Angeles, CA 90089, USA.
}

parameters (Allen, 1986; Keefe et al., 1992; Voss and Allen, 1994). The ear-canal reflectance is subsequently calculated using the ear-canal characteristic impedance, which is inversely proportional to the ear-canal cross-sectional area. Due to anatomical differences between different ear canals, the characteristic impedance needs to be measured or estimated in situ. However, a direct measurement is often impractical because the use of ear molds or computed tomography scans (Egolf et al., 1993; Stinson and Lawton, 1989) substantially increases the time required for a single ear-canal reflectance measurement. In addition, acoustic measurements in the ear canal using an ear probe are affected by evanescent modes (Brass and Locke, 1997; Keefe and Benade, 1981). When the ear canal is modeled using one-dimensional transmission-line theory, these evanescent modes can be approximated by an inertance in series with the plane-wave ear-canal impedance. Last, Nørgaard et al. (2019) showed that the oblique insertion of an ear probe using a standard rubber ear tip introduces a short horn loading between the ear probe and the ear canal. Errors in the ear-canal reflectance may thus result from a mismatch in characteristic impedance or contamination by evanescent modes (Lewis, 2018; Nørgaard et al., 2017a), and from the horn loading of an oblique ear-probe insertion (Nørgaard et al., 2019). Naturally, methods based on a measurement principle fundamentally different from the traditional insert ear probe may be subject to different errors. 
In both clinical applications and the existing research literature, the ear-canal characteristic impedance is either fixed at a constant value [usually corresponding to an earcanal radius of $3.75 \mathrm{~mm},{ }^{1} 3.97 \mathrm{~mm}$ for adults and $2.38 \mathrm{~mm}$ for infants (Keefe and Simmons, 2003), or derived from the utilized ear-tip size (Robinson et al., 2013)], or estimated in situ based on the measured ear-canal impedance. In addition to the fixed characteristic impedance, we identified what we believe to be a representative catalog of methods for measuring the ear-canal reflectance that have been reported and utilized in recent literature. These include the methods of Keefe et al. (1992), Rasetshwane and Neely (2011), Nørgaard et al. (2017a), and Nørgaard et al. (2019) of which the estimation of the ear-canal characteristic impedance is an integral part. We found that this estimate is often sensitive to the residual ear-canal length (i.e., the distance between the ear probe and the tympanic membrane), the insertion angle of the ear-probe relative the ear-canal cross-sectional plane, the available frequency bandwidth of the ear-canal impedance measurement, or a combination of these three principal factors. Consequently, the ear-canal reflectance measured using these existing methods is also sensitive to these three principal factors. Authors have not always reported their specific ear-canalreflectance measurement method and, without a systematic controlled comparison, it is difficult to assess the accuracy of such results and reproduce them. In this paper, we review these existing methods and demonstrate their dependency on the three principal factors in an idealized controlled setting using a uniform occluded-ear simulator.

\section{BACKGROUND}

\section{A. Ear-canal reflectance}

The pressure reflectance $R$ is defined as the ratio of reverse- to forward-propagating pressure components $P_{-}$and $P_{+}$, respectively. Based on the measured ear-canal impedance $Z_{\text {meas }}$ using an ear probe placed in and sealed to the ear canal, the ear-canal reflectance $R$ is traditionally calculated as

$$
R \equiv \frac{P_{-}}{P_{+}} \simeq \frac{Z_{\text {meas }}-Z_{0}}{Z_{\text {meas }}+Z_{0}}
$$

where the characteristic impedance of a lossless ear canal at the position of the ear probe

$$
Z_{0}=\frac{\rho c}{A} .
$$

In this equation, $\rho$ is the density of air, $c$ the speed of sound, and $A$ the ear-canal cross-sectional area at the position of the ear probe. Equation (1) is based on the assumption of a plane-wave sound field in the ear canal and is therefore not exact in a non-uniform ear canal (Farmer-Fedor and Rabbitt, 2002). Nevertheless, the ear-canal reflectance is quantified as a plane-wave reflection coefficient.

\section{Keefe et al. (1992)}

Keefe et al. (1992) estimated the ear-canal characteristic impedance $\hat{Z}_{0}$ as the averaged $\langle\cdot\rangle$ real part $\operatorname{Re}\{\cdot\}$ of the measured ear-canal impedance $Z_{\text {meas }}$,

$$
\hat{Z}_{0}=\left\langle\operatorname{Re}\left\{Z_{\text {meas }}\right\}\right\rangle
$$

Using this estimated ear-canal characteristic impedance $\hat{Z}_{0}$, they calculated the ear-canal reflectance

$$
R=\frac{Z_{\text {meas }}-\hat{Z}_{0}}{Z_{\text {meas }}+\hat{Z}_{0}}
$$

Keefe et al. (1992) reported the accuracy of the estimated characteristic impedance in a set of evaluation tubes of lengths $34.8-51.8 \mathrm{~cm}$ with errors of $3 \%-6 \%$. However, these tube lengths are not representative of any human ear canal. In addition, the derivation of Eq. (3) assumes an infinite frequency bandwidth and this method will presumably be affected by the finite bandwidth of practical measurement systems. The input impedance of longer waveguides exhibit increased resonant behavior within a given frequency range and the absorptive behavior of the tympanic membrane directly influences $\operatorname{Re}\left\{Z_{\text {meas }}\right\}$.

\section{Rasetshwane and Neely (2011)}

Rasetshwane and Neely (2011) estimated the ear-canal characteristic impedance $\hat{Z}_{0}$ by minimizing the time-domain reflectance at time $t=0$. In mathematical terms,

$$
\hat{Z}_{0}=\underset{Z_{0}}{\arg \min }\left|\mathcal{F}^{-1}(t=0)\left[w \frac{Z_{\text {meas }}-Z_{0}}{Z_{\text {meas }}+Z_{0}}\right]\right|,
$$

where $|\cdot|$ denotes the absolute value and $\mathcal{F}^{-1}(t)[\cdot]$ the inverse Fourier transform. Note that Rasetshwane and Neely (2011) multiplied the frequency-domain reflectance by a one-sided low-pass half-width Blackman window $w$. Subsequently, they calculated the ear-canal reflectance using Eq. (4). The synthesis of time-domain transfer functions means that this method may be affected by non-causal effects due to properties of the inverse Fourier transform and due to the Blackman window $w$.

\section{Norgaard et al. (2017a)}

Using the Hilbert transform $\mathcal{H}[\cdot]$, Nørgaard et al. (2017a) estimated the evanescent-modes inertance $\hat{L}$ and ear-canal characteristic impedance $\hat{Z}_{0}$ by minimizing the imaginary and real parts, respectively, and in that order, of the impedance estimation error $\epsilon_{Z}$ of an impedance $Z$,

$$
\epsilon_{Z}=Z-Z_{0}-\mathcal{H}[\operatorname{Im}\{Z\}]-j \mathcal{H}^{-1}[\operatorname{Re}\{Z\}]
$$

calculated from the reflectance. They then calculated the ear-canal reflectance in a manner similar to Eq. (4), but additionally subtracting the impedance of the estimated inertance $j \omega \hat{L}$ from the measured impedance $Z_{\text {meas }}$ to estimate the plane-wave ear-canal impedance

$$
\hat{Z}_{\mathrm{pw}}=Z_{\text {meas }}-j \omega \hat{L} \text {. }
$$

Here, $j$ is the unit imaginary number and $\omega$ the angular frequency. They then calculated the reflectance $R$ using the estimated characteristic impedance $\hat{Z}_{0}$, 


$$
R=\frac{\hat{Z}_{\mathrm{pw}}-\hat{Z}_{0}}{\hat{Z}_{\mathrm{pw}}+\hat{Z}_{0}}
$$

They noted, however, that the method was inaccurate when the acoustic waveguide was non-uniform at the position of the ear probe. Because the Hilbert transform is numerically calculated using forward and inverse Fourier transforms, this method may suffer from issues similar to Rasetshwane and Neely (2011), although here, no window function $w$ is utilized. Instead, the reflectance was truncated to restore differentiability at the transition to the mirrored part of the Hermitian-symmetric spectrum. This truncation frequency can be considered a Nyquist frequency used to synthesize the time-domain reflectance.

\section{Norgaard et al. (2019)}

Nørgaard et al. (2019) reported the dependence of the estimated ear-canal characteristic impedance $\hat{Z}_{0}$ obtained using the method of Nørgaard et al. (2017a) on the ear-probe insertion angle. Non-perpendicular insertion angles introduce a horn loading in front of the ear probe, which they compensated for by calculating the reflectance as

$$
R=\frac{Z_{\text {meas }}-\hat{Z}_{\text {meas }, 0}}{Z_{\text {meas }}+\hat{Z}_{\text {meas }, 0}^{*}} .
$$

Here, the asterisk superscript denotes the complex conjugate and the estimated incident impedance $\hat{Z}_{\text {meas }, 0}$ represents the impedance of the horn loading with a uniform anechoic termination. They estimated the incident impedance $\hat{Z}_{\text {meas }, 0}$ by employing the method of Nørgaard et al. (2017a) at multiple truncation frequencies $\omega_{\mathrm{t}}$ (i.e., deliberately limiting the utilized frequency range), resulting in discrete sets of estimated characteristic impedances $\hat{Z}_{0}\left(\omega_{\mathrm{t}}\right)$ and inertances $\hat{L}\left(\omega_{\mathrm{t}}\right)$. They then fit the polynomials $\hat{Z}_{0}\left(\omega_{\mathrm{t}}\right)=\hat{Z}_{0}^{\prime \prime} \omega_{\mathrm{t}}^{2}+\hat{Z}_{0, \mathrm{ec}}$ and $\hat{L}\left(\omega_{\mathrm{t}}\right)=\hat{L}^{\prime} \omega_{\mathrm{t}}+\hat{L}_{0}$, and estimated the incident impedance

$$
\hat{Z}_{\text {meas }, 0}=3 \hat{Z}_{0}^{\prime \prime} \omega^{2}+\hat{Z}_{0, \mathrm{ec}}+j \omega \hat{L}_{0} .
$$

The ordinate intercepts of the fitted polynomials $\hat{Z}_{0, \text { ec }}$ and $\hat{L}_{0}$ are particularly informative because they represent the lumped-element behavior of the horn loading. In particular, $\hat{Z}_{0, \text { ec }}$ represents an estimate of the characteristic impedance of the ear canal and $\hat{L}_{0}$ approximates the sum of inertances due to the horn loading $L_{\mathrm{horn}}$ and evanescent modes $L_{\mathrm{em}}$, that is, $\hat{L}_{0} \simeq L_{\text {horn }}+L_{\mathrm{em}}$. Finally, they estimated the plane-wave ear-canal impedance as

$$
\hat{Z}_{\mathrm{pw}}=\hat{Z}_{0, \mathrm{ec}} \frac{1+R}{1-R} \text {. }
$$

The number of available truncation frequencies $\omega_{\mathrm{t}}$ depends on the residual ear-canal length and this method may become unstable for shorter ear canals because fewer data points are available for the polynomial fitting process.

\section{B. Stimulus-level and emitted-pressure estimation}

\section{Integrated pressure}

Based on the ear-canal reflectance $R$, Souza et al. (2014) calculated the forward- and reverse-propagating pressure components $P_{+}$and $P_{-}$, respectively, of the total measured ear-probe pressure $P_{\text {meas }}$ from a stimulus supplied by the ear-probe speaker,

$$
\begin{aligned}
& P_{+}=P_{\text {meas }} \frac{R}{1+R}, \\
& P_{-}=P_{\text {meas }} \frac{1}{1+R} .
\end{aligned}
$$

Although the forward pressure $P_{+}$is frequently used for calibrating stimulus level in ear canals, the integrated pressure $P_{\text {int }}$ (Lewis et al., 2009) can be useful for estimating the sound pressure at the termination of a waveguide (e.g., Siegel et al., 2018),

$$
P_{\text {int }}=\left|P_{+}\right|+\left|P_{-}\right|,
$$

and is therefore more intuitive for quantifying errors in stimulus-level calibration with respect to a sound pressure that can be physically measured. The integrated pressure $P_{\text {int }}$ represents the sound pressure at the rigid termination of a uniform waveguide because the forward $P_{+}$and reverse $P_{-}$ pressure components are in phase at that location. We found that the error due the finite termination impedance in a standardized uniform occluded-ear simulator amounts to approximately $0.1 \mathrm{~dB}$. Larger errors can therefore be attributed to errors in the ear-canal reflectance.

\section{Emitted pressure}

Based on the ear-canal reflectance $R$ and the pressure measured using the ear probe from an evoked response from the inner ear $P_{\text {spl }}$ (e.g., an OAE), Charaziak and Shera (2017) calculated the emitted pressure

$$
P_{\mathrm{epl}}=P_{\mathrm{spl}} \frac{1-R R_{\mathrm{s}}}{T\left(1+R_{\mathrm{s}}\right)} .
$$

They calculated the ear-probe source reflectance $R_{\mathrm{S}}$ from the ear-probe source impedance $Z_{\mathrm{s}}$ as obtained during the preliminary Thévenin-equivalent calibration procedure and the estimated ear-canal characteristic impedance $\hat{Z}_{0}$,

$$
R_{\mathrm{s}}=\frac{Z_{\mathrm{s}}-\hat{Z}_{0}}{Z_{\mathrm{s}}+\hat{Z}_{0}}
$$

and the one-way delay $T=e^{-j \omega l / c}$ resulting from the residual ear-canal length $l$. The emitted pressure $P_{\text {epl }}$ represents the inner-ear-evoked pressure at the tympanic membrane were the ear canal and ear probe replaced by an anechoic uniform termination; it is therefore independent of the residual ear-canal length.

\section{THEORY}

Judging from the effects of an oblique ear-probe insertion on the ear-canal reflectance reported by Nørgaard et al. (2019), it is reasonable to expect that the effect of the horn loading needs to be incorporated into the source reflectance $R_{\mathrm{S}}$ as well when calculating the emitted pressure [Eq. (15)]. 
Employing the same theoretical framework as Nørgaard et al. (2019) and assuming that the ear probe is located at the throat, Fig. 1 shows a sketch of a horn loading in front of an anechoic uniform waveguide with characteristic impedance $Z_{0}$, including the forward plane-wave incident throat impedance $Z_{\text {throat, } 0}$. Using a one-dimensional transmission-line model, the forward two-port transfer-matrix elements $a_{i j}$ of the horn loading relate the characteristic impedance $Z_{0}$ to the plane-wave incident throat impedance

$$
Z_{\text {throat }, 0}=\frac{a_{11} Z_{0}+a_{12}}{a_{21} Z_{0}+a_{22}} .
$$

We denote plane-wave impedances as such to indicate a one-dimensional sound field and an absence of higher-order modes, despite the physical waves inside a horn segment being non-planar.

Looking in the reverse direction, Fig. 1 further shows the plane-wave source impedance $Z_{\mathrm{s}}$ and the plane-wave mouth source impedance $Z_{\mathrm{s} \text {,mouth }}$. When operating a nonuniform transmission-line model in reverse, $a_{11} \leftrightarrow a_{22}$, and we can write the plane-wave mouth source impedance

$$
Z_{\mathrm{s}, \text { mouth }}=\frac{a_{22} Z_{\mathrm{s}}+a_{12}}{a_{21} Z_{\mathrm{s}}+a_{11}} .
$$

The desired source reflectance including the horn loading $R_{\mathrm{S}}$ is defined using the plane-wave mouth source impedance $Z_{\mathrm{s} \text {,mouth }}$ and the characteristic impedance of the uniform waveguide $Z_{0}$,

$$
R_{\mathrm{s}}=\frac{Z_{\mathrm{s}, \text { mouth }}-Z_{0}}{Z_{\mathrm{s}, \text { mouth }}+Z_{0}} .
$$

By combining Eqs. (18) and (19), and rearranging,

$$
R_{\mathrm{s}}=\frac{Z_{\mathrm{s}}-\frac{a_{11} Z_{0}-a_{12}}{-a_{21} Z_{0}+a_{22}}}{Z_{\mathrm{s}}+\frac{a_{11} Z_{0}+a_{12}}{a_{21} Z_{0}+a_{22}}} \cdot \frac{-a_{21} Z_{0}+a_{22}}{a_{21} Z_{0}+a_{22}} .
$$

For a lossless transmission line, $a_{11}, a_{22}$, and $Z_{0}$ are real numbers, and $a_{12}$ and $a_{21}$ are imaginary numbers. Using the plane-wave incident throat impedance $Z_{\text {throat, } 0}$ as defined in Eq. (17) and the forward plane-wave incident transfer impedance $Z_{\text {trans, } 0}$ - defined as the ratio of sound pressure at

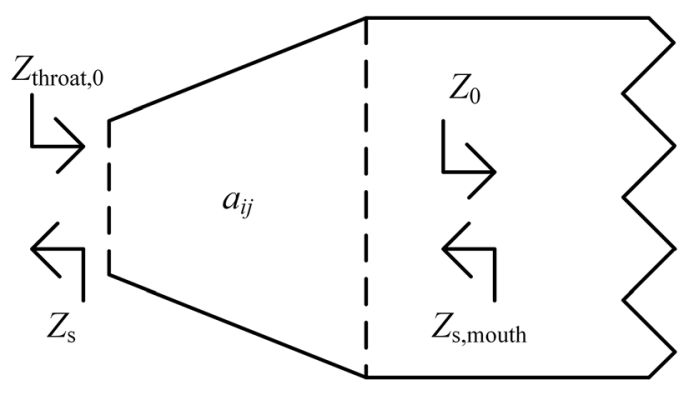

FIG. 1. Sketch of an arbitrary horn segment with two-port transfer-matrix elements $a_{i j}$ terminated by an anechoic uniform waveguide with characteristic impedance $Z_{0}$, including the forward plane-wave incident throat impedance $Z_{\text {throat }, 0}$, and the reverse plane-wave source $Z_{\mathrm{s}}$ and mouth source $Z_{\text {s,mouth }}$ impedances. the mouth $P_{\text {mouth }}$ to volume flow injected at the throat $U_{\text {throat }}$ of the horn segment terminated by $Z_{0}$,

$$
Z_{\text {trans }, 0}=\frac{P_{\text {mouth }}}{U_{\text {throat }}}=\frac{Z_{0}}{a_{21} Z_{0}+a_{22}},
$$

Eq. (20) can be written as

$$
R_{\mathrm{s}}=\frac{Z_{\mathrm{s}}-Z_{\text {throat }, 0}^{*}}{Z_{\mathrm{s}}+Z_{\text {throat }, 0}} \cdot \frac{Z_{\text {trans }, 0}}{Z_{\text {trans }, 0}^{*}} .
$$

That is, we can incorporate the effects of the horn loading onto a reverse incident plane wave on the ear probe using forward plane-wave impedance variables as seen from the ear probe.

\section{METHODS}

\section{A. Source reflectance}

Following Nørgaard et al. (2019), we chose to disregard the delay

$$
\frac{Z_{\text {trans }, 0}}{Z_{\text {trans }, 0}^{*}}=e^{j 2 \angle Z_{\text {trans }, 0}},
$$

in Eq. (22) and calculate the source reflectance $R_{\mathrm{S}}$ using the estimated incident impedance $\hat{Z}_{\text {meas }, 0}$,

$$
R_{\mathrm{s}}=\frac{Z_{\mathrm{s}}-\hat{Z}_{\text {meas }, 0}^{*}}{Z_{\mathrm{s}}+\hat{Z}_{\text {meas }, 0}} .
$$

This formulation is particularly advantageous because the error when calculating the reflectance $R$ using Eq. (9) is the inverse delay (i.e., $Z_{\text {trans }, 0}^{*} / Z_{\text {trans }, 0}$ ), which cancels in the numerator for the product of ear-canal and source reflectances $R R_{\mathrm{s}}$ in Eq. (15). Thus, only the delay error in $R_{\mathrm{s}}$ in the denominator remains.

The estimated incident impedance $\hat{Z}_{\text {meas }, 0}$ using the method of Nørgaard et al. (2019) includes the effect of the evanescent modes inertance $L_{\mathrm{em}}$, i.e.,

$$
\hat{Z}_{\text {meas }, 0} \simeq Z_{\text {throat }, 0}+j \omega L_{\mathrm{em}} .
$$

However, Nørgaard et al. (2019) were unable to determine $L_{\mathrm{em}}$ for an oblique ear-probe insertion and $R_{\mathrm{s}}$ therefore includes an additional error due to the evanescent-modes inertance $L_{\mathrm{em}}$ because the source impedance $Z_{\mathrm{s}}$ is defined in terms of plane waves. This error is negligible if $\left|Z_{\mathrm{s}}\right|$ $\gg\left|\omega L_{\mathrm{em}}\right|$ which is usually the case for ear probes because the probe tube is narrow, typically $\sim 3 \mathrm{~mm}$ effective diameter.

\section{B. Correction of acoustic pressures}

Because any measured pressure $P_{\text {meas }}$ used to estimate stimulus levels is also affected by evanescent modes or a horn loading, Nørgaard et al. (2017a) suggested that the plane-wave ear-canal pressure be estimated as

$$
\hat{P}_{\text {meas }, \mathrm{pw}}=P_{\text {meas }} \frac{\hat{Z}_{\mathrm{pw}}}{Z_{\text {meas }}} .
$$


Equation (26) applies similarly when compensating for the effect of the horn loading using the method of Nørgaard et al. (2019). We then calculated integrated pressure $P_{\text {int }}$ [Eq. (14)] by substituting the estimated plane-wave pressure $\hat{P}_{\text {pw }}$ in place of the measured pressure $P_{\text {meas }}$ in Eqs. (12) and (13). This compensation was performed using the methods of Nørgaard et al. (2017a) and Nørgaard et al. (2019) with $\hat{Z}_{\mathrm{pw}}$ calculated from Eqs. (7) and (11), respectively.

Similarly, the inner-ear-evoked pressure $P_{\text {spl }}$ is affected by the horn loading due to an oblique ear-probe insertion. We thus estimated mouth source impedance

$$
\hat{Z}_{\mathrm{s}, \text { mouth }}=\hat{Z}_{0, \mathrm{ec}} \frac{1+R_{\mathrm{s}}}{1-R_{\mathrm{s}}},
$$

in order to estimate the plane-wave inner-ear-evoked pressure

$$
\hat{P}_{\text {spl,pw }}=P_{\text {spl }} \frac{\hat{Z}_{\mathrm{s}, \text { mouth }}}{Z_{\mathrm{s}}} .
$$

We applied this compensation only using the method of Nørgaard et al. (2019) by substituting $\hat{P}_{\mathrm{spl}, \mathrm{pw}}$ in place of $P_{\mathrm{spl}}$ in Eq. (15).

\section{Measurements and equipment}

The measurements reported in this study were carried out using a FireFace UC sound card (RME Audio, Haimhausen, Germany) controlled through custom-written MATLAB (The MathWorks, Inc., Natick, Massachusetts) software and the third-party utility Playrec. ${ }^{2}$ A Titan-based ear probe (Interacoustics A/S, Middelfart, Denmark) was used, but modified to improve the high-frequency performance and reduce internal crosstalk.

Ear-probe pressures $P_{\text {meas }}$ were measured using the earprobe microphone by supplying a frequency-equalized wideband chirp to the ear-probe speaker to provide a flat earprobe pressure in an anechoic waveguide of radius similar to an adult ear canal. The chirp was played back in the ear probe in phase-locked 2048-sample blocks at a sampling rate of $44.1 \mathrm{kHz}$ which were each recorded using the ear-probe microphone and averaged to reduce noise in the measurements. Prior to the measurements, the ear probe was calibrated to obtain its acoustic Thévenin-equivalent source parameters, the ear-probe source pressure $P_{\mathrm{s}}$ and source impedance $Z_{\mathrm{s}}$, and enable the measurement of acoustic impedance using the calibration method described by Nørgaard et al. (2017b). The cylindrical calibration waveguides were of lengths $l=1.2,1.45,1.75$, and $2 \mathrm{~cm}$, and radius $r=2 \mathrm{~mm}$. Note that when evanescent modes are properly accounted for during the calibration procedure, the source parameters are free from parallel components, and impedance measurements are independent of any mismatch in radius between the calibration waveguides and the acoustic load (Nørgaard et al., 2017b; Nørgaard et al., 2018b). The ear-probe microphone sensitivity was obtained using the microphone calibration method described by Nørgaard et al. (2018a). The overall maximum calibration frequency, and thereby maximum impedance measurement frequency, was $f_{\mathrm{m}}=20 \mathrm{kHz}$. Measured impedances $Z_{\text {meas }}$ were derived from the measured ear-probe pressures $P_{\text {meas }}$ and the Théveninequivalent source parameters,

$$
Z_{\text {meas }}=Z_{\mathrm{s}} \frac{P_{\text {meas }}}{P_{\mathrm{s}}-P_{\text {meas }}} .
$$

We measured the ear-canal impedances $Z_{\text {meas }}$ of a Type 4157 uniform occluded-ear simulator (Brüel \& Kjær Sound \& Vibration A/S, Nærum, Denmark), typically designated a 711 coupler, of radius $r=3.75 \mathrm{~mm}$. Measurements were performed at six approximate residual ear-canal lengths $l=1.5-4 \mathrm{~cm}$ in $0.5-\mathrm{cm}$ increments, using both perpendicular and oblique ear-probe insertions as illustrated in Fig. 2 in a transparent acrylic-glass tube. The ear probe was inserted into the occluded-ear simulator using a standard, mushroomshaped, green, 9-mm rubber ear tip (Sanibel Supply, Middelfart, Denmark), also depicted in Fig. 2. The oblique insertion angle was approximately $50^{\circ}$ relative to the crosssectional plane of the occluded-ear simulator. The various residual ear-canal lengths were achieved by attaching a three-dimensional (3D)-printed part that uniformly extended the occluded-ear simulator to the desired lengths. For each measured ear-canal impedance $Z_{\text {meas }}$ and to additionally simulate the effect of lower frequency-bandwidth measurement systems, we calculated the ear-canal reflectance $R$ using the methods of Keefe et al. (1992) [Eqs. (3) and (4)] using frequency-average regions of $0.1-8$ and $0.1-20 \mathrm{kHz}$, and Rasetshwane and Neely (2011) [Eqs. (4) and (5)] and Nørgaard et al. (2017a) [Eqs. (6)-(8)] using maximum frequencies of $f_{\mathrm{m}}=8$ and $20 \mathrm{kHz}$ for estimating the ear-canal characteristic impedance $\hat{Z}_{0}$. In addition, we calculated the ear-canal reflectance $R$ using the fixed characteristic impedance of the occluded ear-simulator $Z_{0}$ [Eqs. (1) and (2)] and the method of Nørgaard et al. (2019) [Eqs. (9) and (10)]. We implemented each of these methods independently in MATLAB based on the cited references and corresponding equations in Sec. II A. Reported reflectance group delays

$$
\tau_{\mathrm{g}}=-\frac{\mathrm{d} \angle R}{\mathrm{~d} \omega},
$$

were convolved with a 19-point Blackman window to reduce noise. Similar to Voss and Allen (1994) and Robinson et al. (2016), and to normalize reflectance group delays $\tau_{\mathrm{g}}$, we subtracted the round-trip time $\tau_{\mathrm{rt}}$ corresponding to each residual ear-canal length $l$ in the uniform occluded-ear simulator,

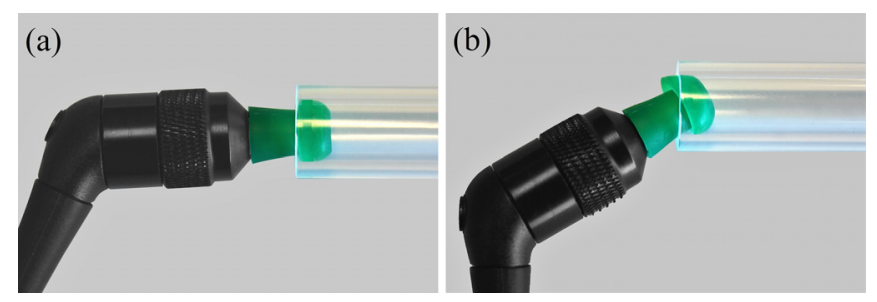

FIG. 2. (Color online) The difference in physical coupling between the ear probe and a transparent acrylic-glass tube of a (a) perpendicular and (b) oblique insertion of the ear probe. Images are taken from Figs. 2(a) and 2(b) of Nørgaard et al. (2019). 


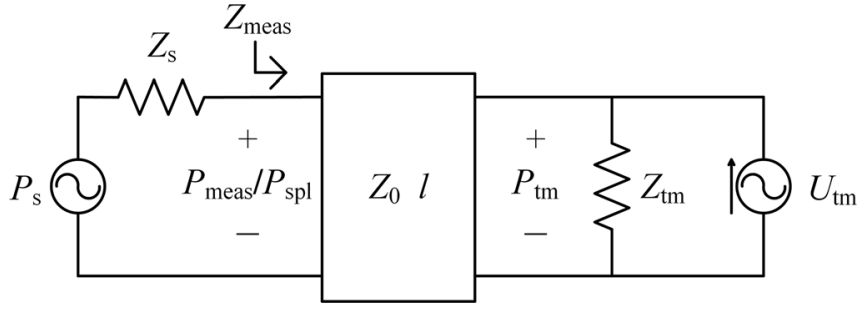

FIG. 3. The electrical analog circuit of an ear probe in a uniform ear canal including dimensional, impedance, and response variables.

$$
\tau_{\mathrm{rt}}=\frac{2 l}{c}
$$

Of course, this compensation for the residual ear-canal length is more complicated in non-uniform ear canals which are often terminated by the tympanic membrane at an oblique angle. We compared the different measurement methods by directly assessing the variation in ear-canal reflectance resulting from the various residual ear-canal lengths, earprobe insertion angles, and measurement frequency bandwidths. Simultaneously, we measured the tympanicmembrane pressure $P_{\mathrm{tm}}$ - that is, the pressure at the reference condenser microphone at the termination of the occluded-ear simulator-and calculated the integrated pressure $P_{\text {int }}$ [Eq. (14)] using the various reflectances $R$.

Subsequent to each measurement, and without physically moving the ear probe, we switched the externally polarized reference condenser microphone at the termination of the occluded-ear simulator to operate as an electro-static speaker. This was achieved by applying a DC-offset sinusoidal voltage to the back plate of the microphone using custom-developed bias and amplification circuitry. By scaling the applied harmonic voltage by $1 / \omega$, the reference microphone now acts as a frequency-independent high-impedance volume-flow source and can be assumed to constitute the Norton-equivalent innerear volume flow $U_{\mathrm{tm}}$, although not completely free from distortion. ${ }^{3}$ We then measured the sound pressure at the ear-probe microphone in response to this inner-ear-evoked excitation $P_{\text {spl }}$ and calculated the emitted pressure $P_{\text {epl }}[$ Eq. (15)] based on the ear-canal reflectances $R$ as obtained using the various considered methods for each residual ear-canal length and earprobe insertion angle. In this paper, we report emitted-pressure magnitudes $\left|P_{\text {epl }}\right|$ and, thus, we set the one-way ear-canal delay $T=1$ in Eq. (15). Based on the emitted pressure $P_{\text {epl }}$ obtained using the fixed characteristic impedance $Z_{0}$ of the occluded-ear-simulator, we manually scaled $U_{\mathrm{tm}}$ such that the magnitude of the ratio $\left|P_{\text {epl }} / U_{\mathrm{tm}} Z_{0}\right|=1$ when $Z_{\mathrm{tm}} \rightarrow \infty$ toward low frequencies. Figure 3 shows the electrical analog of an ear probe placed in a uniform ear-canal transmisison line, including relevant dimensional, impedance, and response variables as described in Secs. II-IV.

\section{RESULTS}

\section{A. Estimated ear-canal characteristic impedance}

Figure 4 shows the estimated ear-canal characteristic impedances $\hat{Z}_{0}$ normalized by the characteristic impedance $Z_{0}$ [Eq. (2)] of the occluded-ear simulator as a function of the residual ear-canal length for the various considered ear-canal-reflectance measurement methods (as indicated in each subfigure), insertion angles (perpendicular and oblique), and measurement frequency bandwidths.

Figures 4(a) and 4(b) show the results obtained using the method of Keefe et al. (1992) with frequency-average regions $0.1-8$ and $0.1-20 \mathrm{kHz}$, respectively, when estimating $\hat{Z}_{0}$ [Eq. (3)]. With $0.1-8 \mathrm{kHz}$ [Fig. 4(a)], we note that $\hat{Z}_{0}$ depends substantially on the residual ear-canal length but not on the ear-probe insertion angle. This is a result of very little resonant behavior of the occluded-ear simulator being included in the measured ear-canal impedance and the halfwave peaks transitioning into the frequency-averaging region when the length increases. With $0.1-20 \mathrm{kHz}$ [Fig. 4(b)], we note that $\hat{Z}_{0}$ mainly depends on insertion angle. This is a result of having adequate resonant behavior included in the frequency average, however, $\hat{Z}_{0}$ is now affected by the oblique ear-probe insertion due to its substantial effect on the measured impedance toward higher frequencies reported by Nørgaard et al. (2019).

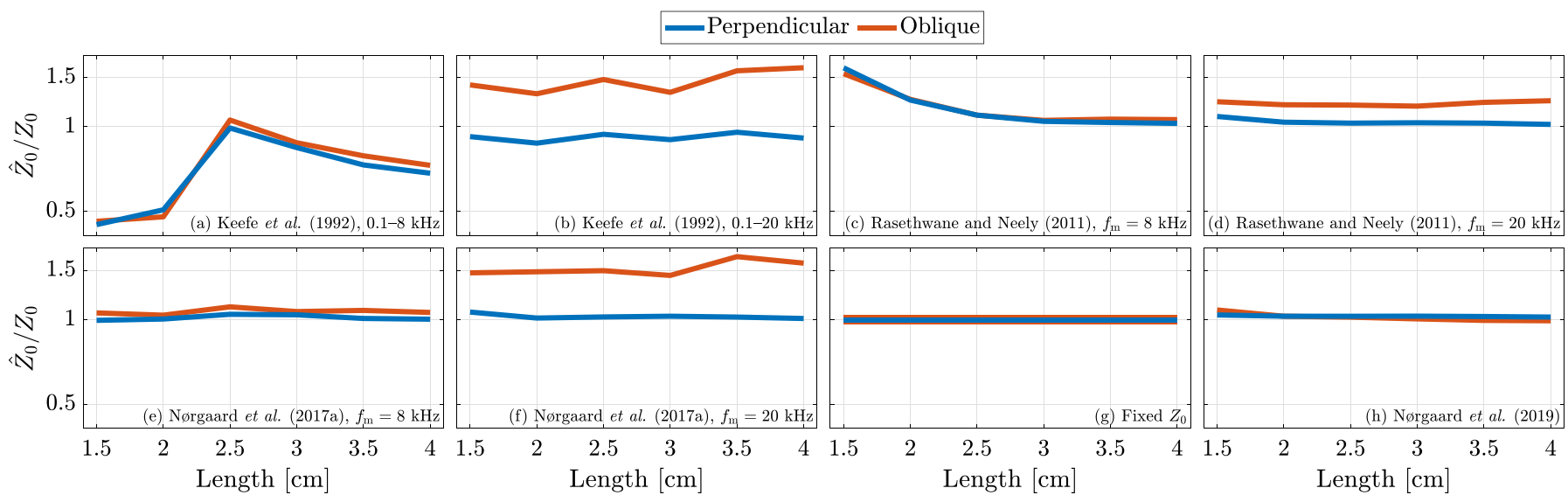

FIG. 4. (Color online) The estimated ear-canal characteristic impedances $\hat{Z}_{0}$ normalized by the characteristic impedance $Z_{0}$ [Eq. (2)] of the occluded-ear simulator as a function of the residual ear-canal length, using perpendicular and oblique ear-probe insertions (see Fig. 2) and the methods of (a),(b) Keefe et al. (1992) [Eq. (3)] with frequency-average regions of (a) $0.1-8$ and (b) $0.1-20 \mathrm{kHz}$, (c),(d) Rasetshwane and Neely (2011) [Eq. (5)] and (e),(f) Nørgaard et al. (2017a) with maximum frequencies (c), (e) $f_{\mathrm{m}}=8$ and (d),(f) $f_{\mathrm{m}}=20 \mathrm{kHz}$, (g) a fixed characteristic impedance, i.e., $\hat{Z}_{0}=Z_{0}$ [Eq. (2)], and (h) Nørgaard et al. (2019), i.e., $\hat{Z}_{0}=\hat{Z}_{0, \text { ec }}$ [see Eq. (10)]. 
Figures 4(c) and 4(d) show the results obtained using the method of Rasetshwane and Neely (2011) with maximum frequencies $f_{\mathrm{m}}=8$ and $20 \mathrm{kHz}$, respectively, when estimating $\hat{Z}_{0}$ [Eq. (5)]. With $f_{\mathrm{m}}=8 \mathrm{kHz}$ [Fig. 4(c)], we note that $Z_{0}$ depends mainly on the residual ear-canal length and not the insertion angle. Due to the low maximum frequency $f_{\mathrm{m}}$, the Blackman window $w$ in Eq. (5) is narrow in frequency. This leads to the convolution of the reflectance with a correspondingly wider window in the time domain. For decreasing residual ear-canal lengths, the positive reflection from the termination of the occluded-ear simulator overlaps increasingly at $t=0$ and causes an increase in $\hat{Z}_{0}$. With $f_{\mathrm{m}}=20 \mathrm{kHz}$ [Fig. 4(d)], we note that $\hat{Z}_{0}$ mainly depends on insertion angle. The larger maximum frequency $f_{\mathrm{m}}$ has widened the frequency-domain window $w$ which results in a narrow window convoluted onto the time-domain reflectance and no longer overlaps at $t=0$ for $l=1.5 \mathrm{~cm}$. In addition, the method is less sensitive to insertion angle compared to Keefe et al. (1992) with $0.1-20 \mathrm{kHz}$ because of the lower weighting given to high frequencies as a result of the frequency-domain windowing $w$ of Eq. (5).

Figures 4(e) and 4(f) show the results obtained using the method of Nørgaard et al. (2017a) with $f_{\mathrm{m}}=8$ and $20 \mathrm{kHz}$, respectively. For $f_{\mathrm{m}}=8 \mathrm{kHz}$ [Fig. $4(\mathrm{e})$ ], we note that this method has little dependence on the insertion angle and no dependence on the residual ear-canal length. This is a result of not utilizing a frequency-domain window $w$ but rather truncating the spectrum to restore differentiability at the truncation frequency as described in Sec. II A 3. When the high-frequency behavior of the oblique ear-probe insertion is not included in the utilized frequency range, the result is a $\hat{Z}_{0}$ close to the true characteristic impedance $Z_{0}$. With $f_{\mathrm{m}}=20 \mathrm{kHz}$ [Fig. 4(f)], we note that this method depends mainly on insertion angle with errors similar to Keefe et al. (1992) with $0.1-20 \mathrm{kHz}$ because the high-frequency behavior of the oblique ear-probe insertion is now included in the frequency range.

Finally, Figs. 4(g) and 4(h) show the results using the fixed characteristic impedance $Z_{0}$ [Eq. (2)] and the method of Nørgaard et al. (2019), respectively. For the fixed $Z_{0}$ [Fig. $4(\mathrm{~g})]$ there is naturally no variation because we plot $\hat{Z}_{0}=Z_{0}$. For Nørgaard et al. (2019) [Fig. 4(h)], we plot $\hat{Z}_{0}=\hat{Z}_{0 \text {,ec }}$ [see Eq. (10)] and note that there is dependence on neither the residual ear-canal length nor on the ear-probe insertion angle. This suggests that the polynomial fitting process described in Sec. II A 4 accurately extracts the lumpedelement behavior of an oblique ear-probe insertion.

\section{B. Ear-canal reflectance}

Figure 5 shows the ear-canal reflectance magnitudes $|R|$ and round-trip-time-normalized reflectance group delays $\tau_{\mathrm{g}}$ $-\tau_{\mathrm{rt}}$ [Eqs. (30) and (31)] of the occluded-ear simulator with the various residual ear-canal lengths, obtained using the measurement methods, ear-probe insertion angles, and frequency-average regions or maximum frequencies $f_{\mathrm{m}}$ indicated in the subfigures.

Using the methods of Keefe et al. (1992) [Eqs. (3) and (4)] with frequency-average regions $0.1-8$ and $0.1-20 \mathrm{kHz}$
[Figs. 5(a) and 5(b), respectively] and Rasetshwane and Neely (2011) [Eqs. (4) and (5)] using $f_{\mathrm{m}}=8$ and $f_{\mathrm{m}}=20 \mathrm{kHz}$ [Figs. 5(c) and 5(d), respectively] the variation in $R$ is a direct result of the variation in the estimated characteristic impedances $\hat{Z}_{0}$ reported in Fig. 4 resulting from the issues described in Sec. V A. In addition, the presence of evanescent modes and the oblique ear-probe insertion introduces errors into these measurements. It is evident from Figs. 5(a)-5(d) that, under these controlled conditions, neither of the methods produces an ear-canal reflectance that is independent of the residual ear-canal length and ear-probe insertion angle.

Using the method of Nørgaard et al. (2017a) [Eqs. (7) and (8)] with $f_{\mathrm{m}}=20 \mathrm{kHz}$ [Fig. 5(f)] there is expectedly a large variation in $R$ between the perpendicular and oblique ear-probe insertions due to the different $\hat{Z}_{0}$ in Fig. 4(f). Additionally, for the oblique insertion, there is also a large dependency of $R$ on the residual ear-canal length despite the constancy of $\hat{Z}_{0}$. However, using Nørgaard et al. (2017a) with $f_{\mathrm{m}}=8 \mathrm{kHz}$ [Fig. 5(e)] there is little variation in $R$ up to $8 \mathrm{kHz}$ between the perpendicular and oblique insertions. This is a result of the low maximum frequency $f_{\mathrm{m}}$ whereby the estimated characteristic impedance $\hat{Z}_{0}$ and inertance $\hat{L}$ are mainly determined from the low-frequency behavior of oblique ear-probe insertion. Therefore, this case corresponds to Eq. (9) (Nørgaard et al., 2019) where the estimated incident impedance $\hat{Z}_{\text {meas }, 0} \simeq \hat{Z}_{0, \text { ec }}+j \omega \hat{L}_{0}$, i.e., without the frequency-squared term $3 \hat{Z}_{0}^{\prime \prime} \omega \omega^{2}$ of Eq. (10).

Using the fixed characteristic impedance $Z_{0}$ [Eqs. (1) and (2), Fig. 5(g)], the variation for the perpendicular earprobe insertions is small and results solely from the effects of evanescent modes. However, for the oblique insertions, the variation is more substantial and a consequence of both the oblique ear-probe insertion and evanescent modes. Finally, using the method of Nørgaard et al. (2019) [Eqs. (9) and (10), Fig. 5(h)], there is little variation in $R$ up to $20 \mathrm{kHz}$ between the perpendicular and oblique ear-probe insertions, and residual ear-canal lengths. Because we used similar perpendicular and oblique ear-probe insertions into the occluded-ear simulator, our estimated incident impedances $\hat{Z}_{\text {meas }, 0}$ were similar to those reported in Fig. 5(e) of Nørgaard et al. (2019).

\section{Stimulus-level calibration and emitted-pressure estimation}

For the perpendicular and oblique ear-probe insertions into the occluded-ear simulator, Figs. 6(a) and 6(b), respectively, show the measured tympanic-membrane pressures $P_{\text {tm }}$ normalized by the measured ear-probe pressures $P_{\text {meas }}$ in response to ear-probe stimulation. When expressed in $\mathrm{dB}$, the ratio can be directly interpreted as the resulting stimulus error. Similarly, for the perpendicular and oblique insertions, Figs. 6(c) and $6(d)$, respectively, show the measured inner-ear-evoked pressures $P_{\text {spl }}$, normalized by the Norton-equivalent inner-ear volume flow $U_{\mathrm{tm}}$ and the characteristic impedance $Z_{0}$ of the occluded-ear simulator. These results, which show stimulus levels varying up to $20 \mathrm{~dB}$ and measured OAE levels varying up to $15 \mathrm{~dB}$ depending on the residual ear-canal length, 


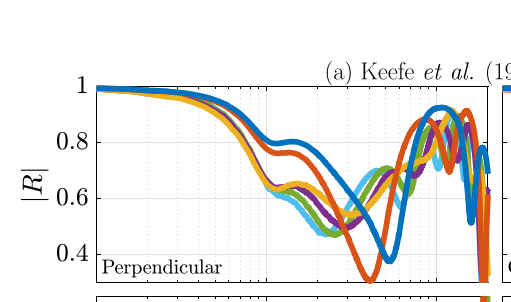

$-1.5 \mathrm{~cm}=2.0 \mathrm{~cm}-2.5 \mathrm{~cm}=3.0 \mathrm{~cm}=3.5 \mathrm{~cm}=4.0 \mathrm{~cm}$
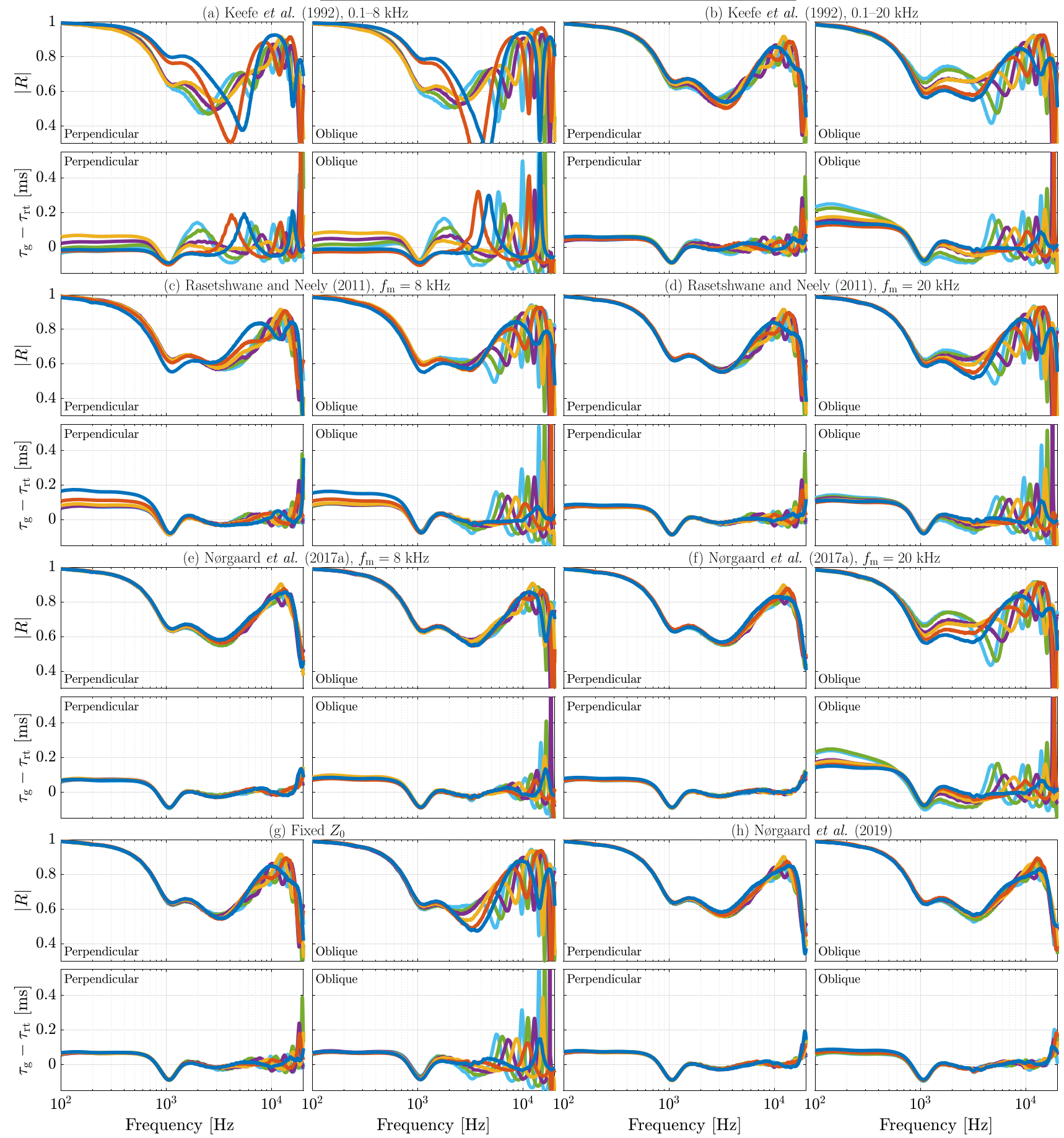

FIG. 5. (Color online) The calculated ear-canal-reflectance magnitudes $|R|$ and round-trip-time-normalized reflectance group delays $\tau_{\mathrm{g}}-\tau_{\mathrm{rt}}$ [Eqs. (30) and (31)] of the occluded-ear simulator at various residual ear-canal lengths, using perpendicular and oblique ear-probe insertions (see Fig. 2) and the methods of (a),(b) Keefe et al. (1992) [Eqs. (3) and (4)] with frequency-average regions of (a) $0.1-8$ and (b) $0.1-20 \mathrm{kHz}$, (c),(d) Rasetshwane and Neely (2011) [Eqs. (4) and (5)] and (e),(f) Nørgaard et al. (2017a) [Eqs. (7) and (8)] with maximum frequencies (c), (e) $f_{\mathrm{m}}=8$ and (d),(f) $f_{\mathrm{m}}=20 \mathrm{kHz}$, (g) a fixed characteristic impedance $Z_{0}$ [Eqs. (1) and (2)], and (h) Nørgaard et al. (2019) [Eqs. (9) and (10)].

illustrate the problem of reproducibility for OAE measurements when no compensation for ear-canal acoustics is performed. Note that the variation due to the oblique ear-probe insertion is negligible compared to the variation resulting from standing waves with different residual ear-canal lengths.

In commercial equipment, it is often standard practice to utilize a so-called "coupler calibration method" for calibrating stimulus levels for OAE measurements. This method is based on the pressure transfer function of an occluded-ear simulator $P_{\mathrm{tm}} / P_{\text {meas }}$ close to the reference input plane (usually corresponding to a residual ear-canal length of $l=1.25 \mathrm{~cm})$ and then assuming that all ear canals have the same pressure transfer function between the ear probe and tympanic membrane. The substantial errors introduced by this calibration method with varying residual ear-canal lengths can be approximately appreciated by noting the 


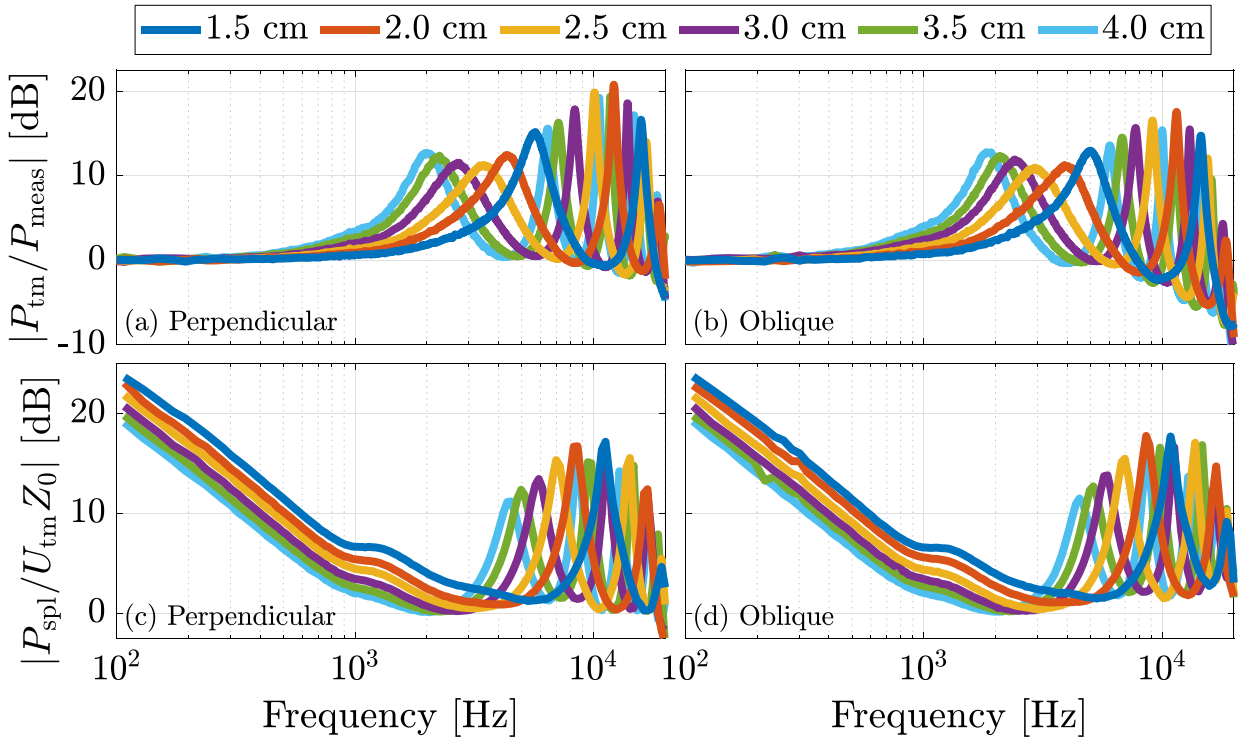

FIG. 6. (Color online) The measured (a),(b) tympanic-membrane pressures $P_{\mathrm{tm}}$ normalized by the ear-probe pressures $P_{\text {meas }}$ in response to ear-probe stimulation and (c),(d) inner-earevoked pressures $P_{\text {spl }}$ normalized by the Norton-equivalent inner-ear volume flow $U_{\mathrm{tm}}$ and the characteristic impedance $Z_{0}$ of the occluded-ear simulator using (a),(c) perpendicular and (b),(d) oblique ear-probe insertions.

discrepancy between the blue line $(1.5 \mathrm{~cm})$ in Fig. 6(a) and the remaining lines in Figs. 6(a) and 6(b). It is evident that this method is not necessarily an improvement over simply utilizing the measured pressure $P_{\text {meas }}$, even if the residual ear-canal length during the calibration is increased (i.e., by taking any other line color in Fig. 6(a) as the reference).

Last, Fig. 7 shows the measured tympanic-membrane pressures $P_{\mathrm{tm}}$ normalized by the calculated integrated pressures $P_{\text {int }}$ [Eq. (14)], and the calculated emitted pressures $P_{\text {epl }}$ [Eq. (15)] normalized by the Norton-equivalent innerear volume flow $U_{\mathrm{tm}}$ and characteristic impedance $Z_{0}$ [Eq. (2)]. Following corresponding panels of Fig. 5, results are shown for the occluded-ear simulator with the various residual ear-canal lengths and obtained using the measurement methods, ear-probe insertion angles, frequencyaverage regions or maximum frequencies $f_{\mathrm{m}}$, and ear-canal reflectances $R$. The errors and variations in $P_{\text {int }}$ and $P_{\text {epl }}$, respectively, correspond generally to the variation in $R$ observed in Fig. 5. However, particularly at low frequencies, $P_{\text {epl }}$ varies directly as a consequence of errors in the estimated characteristic impedance $\hat{Z}_{0}$ in Fig. 4. Similar to Fig. 5, we note that Nørgaard et al. (2017a) with $f_{\mathrm{m}}=8 \mathrm{kHz}$ [Fig. 7(e)] performs well up to $8 \mathrm{kHz}$ and Nørgaard et al. (2019) [Fig. 7(h)] performs reasonably well up to $20 \mathrm{kHz}$, except for a slight divergence between the perpendicular and oblique insertions due to not accounting for the frequency-dependent delay in the oblique ear-probe insertion as described in Sec. IV A. Here, this divergence is approximately $-1 \mathrm{~dB}$ at $10 \mathrm{kHz}$ and $-5 \mathrm{~dB}$ at $20 \mathrm{kHz}$. Notice also from Fig. 7(h) how the tympanic-membrane impedance $Z_{\mathrm{tm}}$ shapes $P_{\text {epl }}$ only marginally (approximately $-2 \mathrm{~dB}$ from 1 to $3 \mathrm{kHz}$ ) when $U_{\mathrm{tm}}$ is constant across frequency because $Z_{\mathrm{tm}} \gg Z_{0}$. This suggests that the characterization of the inner ear as a Nortonrather than Thévenin-equivalent circuit as proposed by Charaziak and Shera (2017) may be preferable.

\section{DISCUSSION}

Although the application of ear-canal reflectance has been widely researched and found useful for a number of hearing-diagnostic applications, comparatively little effort has been put into assessing its accuracy, which is naturally limited by the lack of reliable alternative measurement methods for human ear canals. The results reported in this paper shed light on the sensitivity and variation in existing ear-canal-reflectance measurement methods due to three principal factors that can be accurately controlled in an occluded-ear simulator, namely, the residual ear-canal length, the ear-probe insertion angle, and the measurement frequency bandwidth. We emphasize that these results only represent the variation due to these three principal factors and not due to the inherent non-uniformities of human ear canals and other potential effects not present in the ear simulator, to which the methods may be sensitive to different degrees. Still, the uncertainty of the residual ear-canal length is very much present in reflectance measurements in human ear canals. In addition, recent results of Nørgaard et al. (2019) suggest that the same considerations apply to oblique ear-probe insertions resulting from the misalignment of the ear probe with the cross-sectional plane or the placement of the ear probe at the location of an ear-canal bend. However, oblique ear-probe insertions and the associated errors may be of larger magnitude in human ear canals than what can be obtained using an oblique ear-probe insertion into the uniform occluded-ear simulator as practiced in this paper. It is possible that other probes or types of ear tips (e.g., foam) will be less affected by oblique ear-probe insertions because the expanding foam helps to align the ear probe with the earcanal walls. However, placing the ear probe at the location of a bend of the ear canal may induce a similar effect. Still, more research is needed to systematically assess the implications of oblique ear-probe insertions on ear-canal reflectance measurements.

We emphasize that the results reported using the fixed characteristic impedance $Z_{0}$ [Figs. $4(\mathrm{~g}), 5(\mathrm{~g})$, and $7(\mathrm{~g})$ ] do not capture the variation in ear-canal reflectance due to the natural anatomical cross-sectional variation in ear-canal geometry, but only the variation due to the presence of evanescent modes and the oblique ear-probe insertion for the specific cross-sectional area of the occluded-ear simulator. 


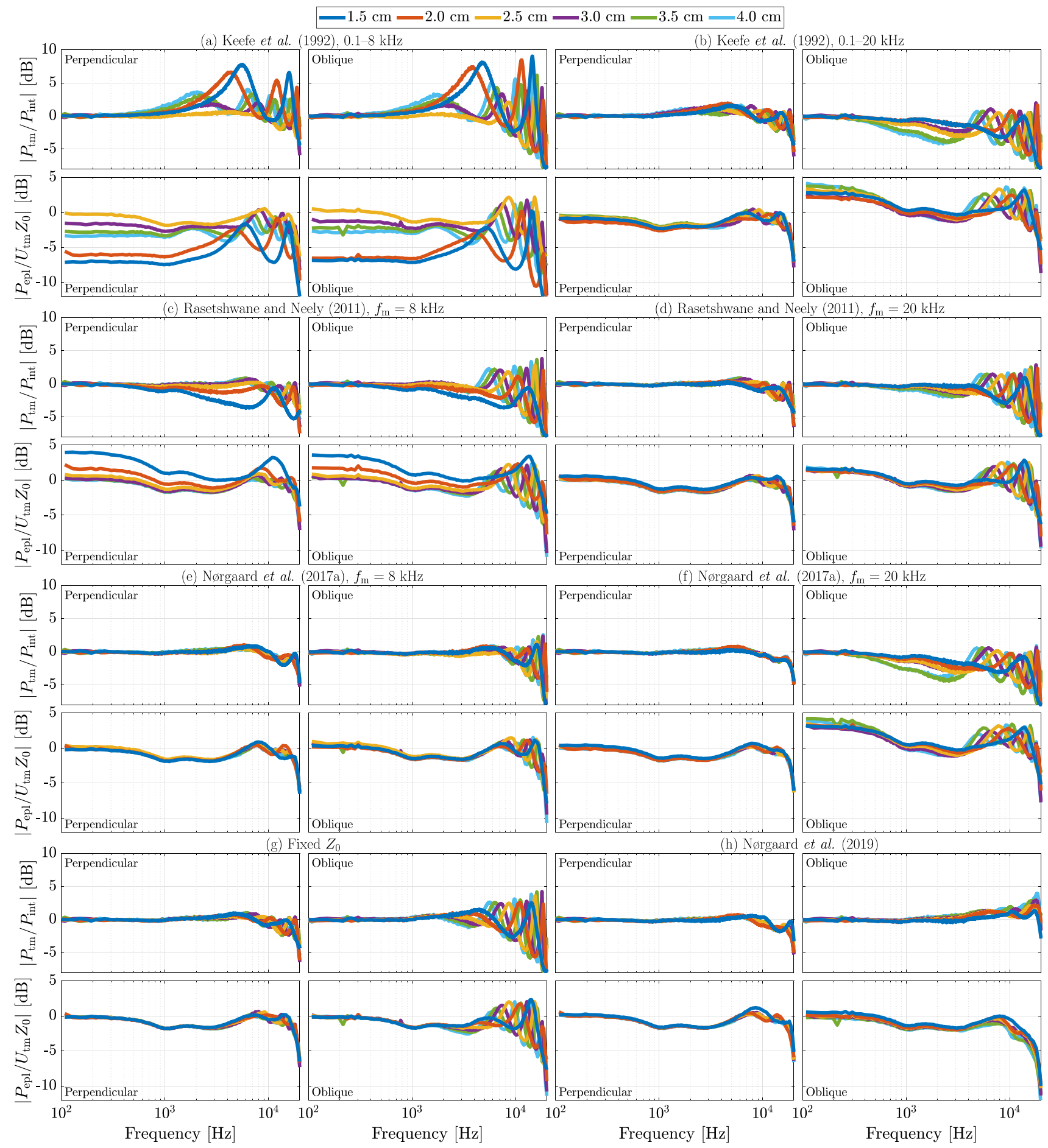

FIG. 7. (Color online) The measured tympanic-membrane pressures $P_{\mathrm{tm}}$ normalized by the calculated integrated pressures $P_{\text {int }}$ [Eq. (14)], and the calculated emitted pressures $P_{\text {epl }}$ [Eq. (15)] normalized by the Norton-equivalent inner-ear volume flow $U_{\text {tm }}$ and characteristic impedance $Z_{0}$ [Eq. (2)] of the occluded-ear simulator at various residual ear-canal lengths, using perpendicular and oblique ear-probe insertions (see Fig. 2) obtained using the ear-canal reflectances $R$ of corresponding panels in Fig. 5, i.e., the methods of (a),(b) Keefe et al. (1992) using frequency-average regions of (a) $0.1-8$ and (b) $0.1-20 \mathrm{kHz}$, (c),(d) Rasetshwane and Neely (2011) and (e),(f) Nørgaard et al. (2017a) [Eq. (26)] using maximum frequencies (c),(e) $f_{\mathrm{m}}=8 \mathrm{and}$ (d),(f) $f_{\mathrm{m}}=20 \mathrm{kHz},(\mathrm{g})$ a fixed characteristic impedance $Z_{0}$, and (h) Nørgaard et al. (2019) [Eqs. (24), (26), and (28)].

Employing a fixed $Z_{0}$ is in fact the approach used in currently available commercial equipment [i.e., the HearID and OtoStat (Mimosa Acoustics, Inc., Champaign, Illinois), and the Titan (Interacoustics A/S, Middelfart, Denmark)]. We therefore expect that the variation in ear-canal reflectance can be even larger using this approach when variations in ear-canal geometry are present. In addition, the contribution from evanescent modes can differ substantially depending on the probe-tube geometry, and the geometrical mismatch between probe-tube and waveguide geometry (Keefe and Benade, 1981; Fletcher et al., 2005; Siegel et al., 2018). Therefore, different measurement systems may be differently affected by evanescent modes. Because our results are obtained in a standardized occluded-ear simulator based on 
an adult ear, they are not representative of the variation due to the three principal factors in younger or infant ears. We also emphasize that, because the ear probe was calibrated just before the measurements, the reported results do not represent possible variation and errors due to instability and drift in the ear-probe calibration over time or due to external effects. The reflectance group delay has occasionally been used as a means of estimating the residual ear-canal length (e.g., Keefe et al., 2015; Robinson et al., 2013; Robinson et al., 2016). Our results in Fig. 5 demonstrate how our three principal factors may affect this length estimate which is only valid in regions where the round-trip-time-normalized group delay $\tau_{\mathrm{g}}-\tau_{\mathrm{rt}} \approx 0$.

By necessity, our study employed only a subset of the measurement configurations (i.e., residual ear-canal lengths and ear-probe insertion angles) and parameters (measurement frequency bandwidths) available. As evident from the results, some ear-canal-reflectance measurement methods are sensitive to either the residual ear-canal length or earprobe insertion angle and we were unable to find an optimal compromise in maximum frequency for these methods with a minimum dependency on either variable. Of course, the reported results should be interpreted with a desired measurement accuracy in mind. Therefore, the results are useful as guidelines for choosing an appropriate method in a given context, but also for judging the potential impact of the three principle factors on the measurement accuracy in the existing literature. Greater reproducibility comes at the price of employing more advanced methods. For example, the method of Keefe et al. (1992) is simple, robust, and easy to implement; conversely the method of Nørgaard et al. (2019) is more complex. Although the method of Nørgaard et al. (2017a) appears to perform well using the maximum frequency $f_{\mathrm{m}}=8 \mathrm{kHz}$, its accuracy may be limited in ear canals if the horn loading of the oblique ear-probe insertion is longer or more severe than what can be achieved in a uniform occluded-ear simulator because the effects of the oblique insertion occur at lower frequencies.

Ear-canal reflectance measurements have been utilized both in research and clinical settings for a number of years. Frustratingly, manufacturers and researchers do not always clearly or consistently report the specific method used for measuring the ear-canal reflectance. Due to the substantial variation observed in the ear-canal reflectance in this paper, this naturally raises largely unanswerable questions about the accuracy of some published results. Going forward, however, our study provides guidance on which methods should be avoided depending on the desired reproducibility and available bandwidth of the utilized measurement system. We strongly urge manufacturers and researchers to clearly report and describe the methods used to perform ear-canal reflectance measurements. Details of the methods are essential for assessing the sensitivity to the residual ear-canal length and ear-probe insertion angle, and for reproducing the results.

\section{CONCLUSION}

In this paper, we have assessed the sensitivity of existing ear-canal-reflectance measurement methods to the residual ear-canal length, the ear-probe insertion angle, and the measurement frequency bandwidth in an idealized controlled setup. In addition, we have investigated the associated errors and variation in estimating the pressure at the tympanic membrane and the emitted pressure, respectively. We found that the method of Nørgaard et al. (2017a) using a maximum frequency $f_{\mathrm{m}}=8 \mathrm{kHz}$ provided good reproducibility up to $8 \mathrm{kHz}$ and the method of Nørgaard et al. (2019) provided good reproducibility up to $20 \mathrm{kHz}$, although the latter was affected by a slight divergence when estimating the emitted pressure toward higher frequencies for the oblique ear-probe insertions. Our results also suggest that the method of assuming a fixed characteristic impedance, as practiced in commercial clinical equipment, should be avoided in adult ears. However, those of our results demonstrating substantial reproducibility in and an independence of the ear-canal reflectance on the three principal factors cannot serve directly as a validation of measurement accuracy of the ear-canal reflectance.

\section{ACKNOWLEDGMENTS}

This research was funded by the Innovation Fund Denmark, Grant No. 5189-00100B (K.R.N.), the National Institutes of Health, Grant Nos. K99 DC016906 (K.K.C.) and R01 DC003687 (C.A.S.), and Interacoustics A/S.

\footnotetext{
${ }^{1}$ See www.mimosaacoustics.com/resources/faq_mepa_tymp.html (Last viewed 1 August 2019).

${ }^{2}$ www.playrec.co.uk (Last viewed 25 July 2019).

${ }^{3}$ The microphone distorts slightly when operated as an electro-static speaker because it only has a charged back plate and no oppositely charged front plate to equalize the non-linear voltage-squared relationship between the applied voltage and force between capacitor plates.
}

Allen, J. B. (1986). "Measurement of eardrum acoustic impedance," in Peripheral Auditory Mechanisms, edited by J. Allen, J. Hall, A. Hubbard, S. Neely, and A. Tubis (Springer-Verlag, New York), pp. 44-51.

Brass, D., and Locke, A. (1997). "The effect of the evanescent wave upon acoustic measurements in the human ear canal," J. Acoust. Soc. Am. 101, 2164-2175.

Charaziak, K. K., and Shera, C. A. (2017). "Compensating for ear-canal acoustics when measuring otoacoustic emissions," J. Acoust. Soc. Am. 141, 515-531.

Egolf, D. P., Nelson, D. K., Howell, H. C., and Larson, V. D. (1993). "Quantifying ear-canal geometry with multiple computer-assisted tomographic scans," J. Acoust. Soc. Am. 93, 2809-2819.

Ellison, J. C., Gorga, M., Cohn, E., Fitzpatrick, D., Sanford, C. A., and Keefe, D. H. (2012). "Wideband acoustic transfer functions predict middle-ear effusion," Laryngoscope 122, 887-894.

Farmer-Fedor, B. L., and Rabbitt, R. D. (2002). "Acoustic intensity, impedance and reflection coefficient in the human ear canal," J. Acoust. Soc. Am. 112, 600-620.

Feeney, M. P., and Keefe, D. H. (2001). "Estimating the acoustic reflex threshold from wideband measures of reflectance, admittance, and power," Ear Hear. 22, 316-332.

Fletcher, N. H., Smith, J., Tarnopolsky, A. Z., and Wolfe, J. (2005). "Acoustic impedance measurements-correction for probe geometry mismatch,” J. Acoust. Soc. Am. 117, 2889-2895.

Keefe, D. H., and Benade, A. H. (1981). "Impedance measurement source and microphone proximity effects,” J. Acoust. Soc. Am. 69, 1489-1495.

Keefe, D. H., Folsom, R. C., Gorga, M. P., Vohr, B. R., Bulen, J. C., and Norton, S. J. (2000). "Identification of neonatal hearing impairment: Earcanal measurements of acoustic admittance and reflectance in neonates," Ear Hear. 21, 443-461.

Keefe, D. H., Hunter, L. L., Feeney, M. P., and Fitzpatrick, D. F. (2015). "Procedures for ambient-pressure and tympanometric tests of aural acoustic reflectance and admittance in human infants and adults," J. Acoust. Soc. Am. 138, 3625-3653. 
Keefe, D. H., Ling, R., and Bulen, J. C. (1992). "Method to measure acoustic impedance and reflection coefficient," J. Acoust. Soc. Am. 91, $470-485$.

Keefe, D. H., Sanford, C. A., Ellison, J. C., Fitzpatrick, D. F., and Gorga, M. P. (2012). "Wideband aural acoustic absorbance predicts conductive hearing loss in children," Int. J. Audiol. 51, 880-891.

Keefe, D. H., and Simmons, J. L. (2003). "Energy transmittance predicts conductive hearing loss in older children and adults," J. Acoust. Soc. Am. 114, 3217-3238.

Lewis, J. D. (2018). "The area discontinuity between probe and ear canal as a source of power-reflectance measurement-location variability," J. Acoust. Soc. Am. 143, 1106-1116.

Lewis, J. D., McCreery, R. W., Neely, S. T., and Stelmachowicz, P. G. (2009). "Comparison of in-situ calibration methods for quantifying input to the middle ear," J. Acoust. Soc. Am. 126, 3114-3124.

McCreery, R. W., Pittman, A., Lewis, J., Neely, S. T., and Stelmachowicz, P. G. (2009). "Use of forward pressure level to minimize the influence of acoustic standing waves during probe-microphone hearing-aid verification," J. Acoust. Soc. Am. 126, 15-24.

Merchant, G. R., Röösli, C., Niesten, M. E. F., Hamade, M. A., Lee, D. J., McKinnon, M. L., Ulku, C. H., Rosowski, J. J., Merchant, S. N., and Nakajima, H. H. (2014). "Power reflectance as a screening tool for the diagnosis of superior semicircular canal dehiscence," Otol. Otolaryngol. 36, 172-177.

Nørgaard, K. R., Fernandez-Grande, E., and Laugesen, S. (2017a). "Compensating for evanescent modes and estimating characteristic impedance in waveguide acoustic impedance measurements," J. Acoust. Soc. Am. 142, 3497-3509.

Nørgaard, K. R., Fernandez-Grande, E., and Laugesen, S. (2017b). "Incorporating evanescent modes and flow losses into reference impedances in acoustic Thévenin calibration,” J. Acoust. Soc. Am. 142, 3013-3024.

Nørgaard, K. R., Fernandez-Grande, E., and Laugesen, S. (2018a). "A coupler-based calibration method for ear-probe microphones," J. Acoust. Soc. Am. 144, 2294-2299.

Nørgaard, K. R., Fernandez-Grande, E., and Laugesen, S. (2019). "Compensating for oblique ear-probe insertions in ear-canal reflectance measurements," J. Acoust. Soc. Am. 145, 3499-3509.
Nørgaard, K. R., Neely, S. T., and Rasetshwane, D. M. (2018b). "Quantifying undesired parallel components in Thévenin-equivalent acoustic source parameters," J. Acoust. Soc. Am. 143, 1491-1503.

Piskorski, P., Keefe, D. H., Simmons, J. L., and Gorga, M. P. (1999). "Prediction of conductive hearing loss based on acoustic ear-canal response using a multivariate clinical decision theory," J. Acoust. Soc. Am. 105, 1749-1764.

Rasetshwane, D. M., and Neely, S. T. (2011). "Inverse solution of ear-canal area function from reflectance," J. Acoust. Soc. Am. 130, 3873-3881.

Robinson, S. R., Nguyen, C. T., and Allen, J. B. (2013). "Characterizing the ear canal acoustic reflectance and impedance by pole-zero fitting," Hear. Res. 301, 168-182.

Robinson, S. R., Thompson, S., and Allen, J. B. (2016). "Effects of negative middle ear pressure on wideband acoustic immittance in normal-hearing adults," Ear Hear. 37, 452-464.

Scheperle, R. A., Goodman, S. S., and Neely, S. T. (2011). "Further assessment of forward pressure level for in situ calibration," J. Acoust. Soc. Am. 130, 3882-3892.

Scheperle, R. A., Neely, S. T., Kopun, J. G., and Gorga, M. P. (2008). "Influence of in situ, sound-level calibration on distortion-product otoacoustic emission variability," J. Acoust. Soc. Am. 124, 288-300.

Siegel, J. H., Nørgaard, K. R., and Neely, S. T. (2018). "Evanescent waves in simulated ear canals: Experimental demonstration and method for compensation,” J. Acoust. Soc. Am. 144, 2135-2146.

Souza, N. N., Dhar, S., Neely, S. T., and Siegel, J. H. (2014). "Comparison of nine methods to estimate ear-canal stimulus levels," J. Acoust. Soc. Am. 136, 1768-1787.

Stinson, M. R., and Lawton, B. W. (1989). "Specification of the geometry of the human ear canal for the prediction of sound-pressure level distribution," J. Acoust. Soc. Am. 85, 2492-2503.

Voss, S. E., and Allen, J. B. (1994). "Measurement of acoustic impedance and reflectance in the human ear canal," J. Acoust. Soc. Am. 95, 372-384.

Withnell, R. H., Jeng, P. S., Waldvogel, K., Morgenstein, K., and Allen, J. B. (2009). "An in situ calibration for hearing thresholds," J. Acoust. Soc. Am. 125, 1605-1611. 